\title{
Diseño de celdas de manufactura
} considerando el balanceo de las cargas de trabajo con algoritmos genéticos Design of manufacturing cells considering workload balancing with genetic algorithms

Conception de cellules de manufacture utilisant le balancement des charges de travail comme algorithmes génétiques

Design de células de manufatura considerando o equilíbrio das cargas de trabalho com algoritmos genéticos

Edisson Suesca M.* Milena Bermúdez C.** Camilo Mejía M.*** Luis Armando Cobo C.**** Leonardo Rodríguez U.*****

Fecha de recepción: 9 de febrero

Fecha de aprobación: 8 de marzo

Pp. $89-122$

* Ingeniero industrial, Fundación Universitaria Los Libertadores. Profesional Asistente de Gerencia de proyectos GEOCOL CONSULTORES S.A.

** Ingeniera industrial, Fundación Universitaria Los Libertadores. Profesional Gestión Humana GEOCOL CONSULTORES S.A.

*** Ingeniero mecánico,Universidad Nacional de Colombia; magíster en Ingeniería Mecánica, Universidad Nacional de Colombia. Profesor Asociado; Facultad de Ingeniería Universidad EAN.

${ }^{* * * *} \mathrm{PhD}$ en Génie Informatique, École Polytechnique de Montréal; doctor en Ingeniería, Universidad de Los Andes; magíster en ingeniería de Sistemas, Universidad de Los Andes, ingeniero de sistemas, Universidad de Los Andes; profesor titular Facultad de Ingeniería Universidad EAN.

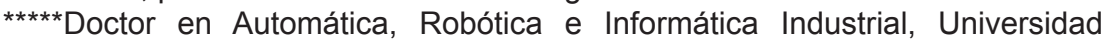
Politécnica de Valencia; máster en Tecnología Energética para el Desarrollo Sostenible,Universidad Politécnica de Valencia; ingeniero mecatrónico Universidad de San Buenaventura; máster en Automática e Informática Industrial, Universidad Politécnica de Valencia. Profesor titular, Facultad de Ingeniería, Universidad EAN. 


\section{RESUMEN}

Este documento presenta la aplicación de algoritmos genéticos al proceso de diseño de sistemas de manufactura celular, solucionando de forma simultánea la formación de las celdas de manufactura y el balanceo de las cargas de trabajo, mediante la maximización de la eficacia de agrupamiento y la eficiencia de línea. El proceso realizado incluyó la formulación del modelo matemático que posteriormente fue implementado y resuelto en Matlabß. La medición del desempeño de esta propuesta se evaluó con problemas prueba, para los cuales se obtuvieron soluciones óptimas y el promedio de las réplicas realizadas estuvo muy cerca del valor óptimo, con una desviación porcentual respecto a este inferior al $1.2 \%$. Además, la configuración de las soluciones obtenidas logra una adecuada agrupación de las celdas y un flujo continuo de las piezas debido a la reducción de los cuellos de botella.

\section{Palabras clave}

Celdas de manufactura, formación de celdas, balanceo de cargas de trabajo, algoritmos genéticos.

\section{ABSTRACT}

This paper presents the application of genetic algorithms to the design process of cellular manufacturing systems, solving the formation of manufacturing cells and workload balancing simultaneously, by maximizing the effectiveness of clustering and the efficiency of the line. The process that was performed included the development of the mathematical model which was subsequently implemented and resolved at Matlab ${ }^{\circledR}$. The performance measurement of this proposal was evaluated with test problems, for which optimal solutions were obtained and the average of replicas that were made was very close to the optimum value, with a deviation percentage of less than $1.2 \%$ regarding the last one obtained. In addition, the configuration of the obtained solutions reach adequate cell clustering and a continuous flow of parts due to bottleneck reduction.

\section{KEYWORDS}

Manufacturing cells, cell formation, balancing workloads, genetic algorithms. 


\section{RÉSUMÉ}

Ce document présente l'application d'Algorithmes Génétiques au processus de conception de systèmes de manufacture cellulaire résolvant de manière simultanée la formation des cellules de manufacture et le balancement des charges de travail grâce à la maximisation de l'efficacité de regroupement et l'efficacité de ligne. Le processus réalisé inclut la formulation du modèle mathématique ayant été mis en place et résolu dans Matlab®. La mesure de la performance de cette proposition a été évaluée par des problèmes d'essai type pour lesquels des solutions optimums ont été obtenues, et la moyenne des répliques réalisées fut très proche de la valeur optimum, d'une déviation inférieure à 1,2\%. La configuration des solutions obtenues atteint de plus un regroupement approprié des cellules et un flux continu des pièces grâce à la réduction des engorgements.

\section{MOTS CLEFS}

Cellules de manufacture, formation de cellules, balancement de charges de travail, algorithmes génétiques..

\section{RESUMO}

Este trabalho apresenta a aplicação de Algoritmos Genéticos ao processo de desenho de sistemas de manufatura celular, resolvendo simultaneamente a formação de células de manufatura e o equilíbrio das cargas de trabalho, maximizando a eficácia do agrupamento e eficiência da linha. O processo executado incluiu o desenvolvimento do modelo matemático, que foi subsequentemente aplicado e resolvido em Matlab®. A medição do desempenho desta proposta foi avaliada com problemas de teste para os quais se obtiveram as melhores soluções e a média das réplicas realizadas foi muito próxima do valor óptimo, com uma porcentagem de desvio inferior a 1,2\%. Além disso, a configuração das soluções obtidas atingiu um agrupamento adequado das células e um fluxo contínuo de peças devido a redução dos pontos de estrangulamento.

\section{Palavras-chave}

Células de manufatura, a formação de células, equilíbrio das cargas de trabalho, algoritmos genéticos. 


\section{Introducción}

os sistemas de producción actuales se ven amenazados constantemente por las dinámicas de la globalización y las condiciones cambiantes del mercado. Esta situación obliga a las organizaciones a tener arquitecturas productivas más flexibles y eficientes, que puedan responder de una forma ágil a cambios en los productos y procesos como lo hacen las celdas de manufactura.

Las celdas de manufactura son sistemas productivos que permiten descomponer una fábrica en mini fábricas o celdas las cuales son unidades más simples donde se fabrican familias de partes. Este cambio facilita enormemente la administración y el control del sistema de producción, y al mismo tiempo lo hace más flexible con lo cual puede responder de una manera más ágil a los cambios que se pueden dar en el mercado. Adicionalmente, también reducen el transporte y la manipulación de los materiales en proceso (Mejía, Lara, y Nieto, 2010), debido a que una parte no debe moverse por toda la planta sino únicamente al interior de la celda; estas y otras ventajas han llevado a la difusión e implementación de las celdas de manufactura en muchas partes del mundo. Se trata de una de las arquitecturas productivas más deseables para sistemas de manufactura flexibles, ya que representa una de las aplicaciones de manufactura esbelta (Sundar, Balaji, \& Kumar, 2014)(Romero, Mejía, y Torres, 2015).

En este contexto es importante también considerar que el sistema de manufactura debe mantener un flujo continuo de los materiales en proceso que minimice las demoras y los inventarios, lo cual se logra con un adecuado balanceo de las cargas de trabajo de las máquinas y/o puestos de trabajo al interior de las celdas de manufactura. Además, el diseño de sistemas de manufactura celular generalmente se ha enfocado 
en el proceso de formación de las celdas de manufactura, teniendo como objetivo principal la conformación de las celdas de manufactura y las familias de partes. Sin embargo, esto no garantiza tener una operación fluida, por lo tanto, es necesario integrar a este proceso el balanceo de las cargas de trabajo para lograr sistemas productivos más eficientes.

Teniendo en cuenta lo anterior, este trabajo presenta el diseño óptimo de sistemas de manufactura celular mediante la solución simultánea de la formación de las celdas de manufactura y el balanceo de las cargas de trabajo empleando Algoritmos Genéticos (AG).

A continuación se describe el proceso desarrollado, iniciando con una introducción a la formación de las celdas de manufactura y el balanceo de las cargas de trabajo; luego, se presenta el modelo matemático propuesto, la estructura del algoritmo genético empleado con la codificación de las soluciones y los operadores implementados; posteriormente, la metodología y el desarrollo experimental implementado; y se finaliza con la presentación de los resultados obtenidos y las conclusiones. 


\section{Formación de celdad de manufactura}

E I proceso de formación de celdas y familias de partes, consiste En agrupar las máquinas en celdas a partir de las similitudes en diseño y procesos de fabricación de las partes que estas procesan, las cuales conforman las familias de partes. De esta forma cada familia tiene asignada una celda de manufactura.

El primer paso para implementar celdas de manufactura es la formación de las celdas para lo cual existen diversos métodos como lo describen Selim, Askin, \& Vakharia (1998), y Papaioannou \& Wilson (2010) quienes presentan una completa taxonomía de estos métodos. Entre estos resaltan las técnicas meta heurísticas como Recocido Simulado (Wu, Chang, \& Chung, 2008), Optimización por Colonia de Hormigas (Spiliopoulos \& Sofianopoulou, 2006) y Algoritmos Genéticos (Saeedi, 2010), tal como lo describen Papaioannou \& Wilson (2010). Entre estas técnicas se destacan los Algoritmos Genéticos ( $A G)$, que son algoritmos de búsqueda basados en la mecánica de la selección natural y la genética, los cuales logran un buen desempeño en la formación de las celdas como lo demuestra el trabajo de Saeedi (2010), quien plantea un modelo matemático para el problema de la formación de celdas de manufactura y compara los resultados que obtuvo solucionando su modelo con Recocido Simulado, Optimización por Colonia de Hormigas y Algoritmos Genéticos, siendo estos últimos los que tuvieron el mejor desempeño. Entre las primeras propuestas que implementan algoritmos genéticos en la formación de celdas de manufactura se encuentra el trabajo de Venugopal \& Narendran (1992); otros trabajos con resultados interesantes que continuaron por esta línea se encuentra en Billo, Bidanda, \& Tate (1996) y Rajagopalan \& Fonseca (2005). 
De forma paralela a los métodos de formación de la celdas de manufactura es necesario considerar la calidad del agrupamiento que generan para lo cual se han creado diversas métricas como se presenta en Sarker (2001). Estas métricas, por su función principal, son muy útiles para emplearlas como función objetivo, ya que en este esquema de trabajo el proceso de optimización realiza la búsqueda de la mejor solución guiado por la métrica de agrupamiento que actúa como función objetivo. Siguiendo este esquema, en el presente trabajo se empleó la eficacia de agrupamiento $(\Gamma)$, la cual fue planteada por Suresh Kumar \& Chandrasekharan (1990). Esta, presenta una estructura simple que permite lograr celdas con un buen agrupamiento de las máquinas y partes en cada celda, que además logra minimizar los movimientos entre celdas que generan incrementos en los costos de transporte y manipulación de los materiales.

\section{Balanceo de cargas de trabajo}

$\Delta$ dicionalmente al proceso de formación de las celdas es indispensable considerar el balanceo de las cargas de trabajo para lograr un sistema de manufactura esbelto (Sundar et al., 2014), en el cual las celdas tengan un flujo constante que disminuya el lead time y el inventario en proceso. En términos generales, el balanceo de las cargas de trabajo consiste en equilibrar el tiempo total de las operaciones o procesos asignados a cada puesto de trabajo o máquina de la celda. Este concepto tiene origen en las líneas de ensamble de los sistemas de producción por producto, los cuales manejan altos volúmenes de producción de productos estandarizados (Becker \& Scholl, 2006). Una línea de ensamble está conformada por estaciones 
de trabajo ubicadas a lo largo de una banda transportadora o un mecanismo para el transporte y manejo de materiales similar, el cual mueve las piezas entre las estaciones de trabajo para que en cada una de estas les realicen las operaciones o procesos que se les han asignado en un tiempo que no debe superar el tiempo ciclo, el cual es el máximo tiempo disponible con el que cuenta cada estación de trabajo para realizar las operaciones que le han sido asignadas (Becker \& Scholl, 2006). El balanceo de cargas de trabajo generalmente se plantea de tres formas tal como está descrito en Battaïa \& Dolgui (2013). La primera, es minimizando el número de estaciones de trabajo; la segunda, minimizando el tiempo ciclo de línea; y la tercera, es maximizando la eficiencia de la línea $(\Gamma)$, con lo cual se busca reducir al mínimo la diferencia entre el tiempo ciclo de cada máquina y el cociente de la sumatoria de los tiempos de operación de cada parte en cada máquina entre la cantidad de máquinas de cada tipo. Esta última fue la forma implementada en este trabajo. Sin embargo, existen otros planteamientos y formas como se ha abordado este problema que pueden consultarse en Becker \& Scholl (2006) y Battaïa \& Dolgui (2013).

\section{Modelo matemático}

-onsiderando el objetivo de diseñar sistemas de manufactura celular que optimicen el agrupamiento de las máquinas y partes en las celdas y familias, y logren un adecuado balanceo de las cargas de trabajo, se plantea el siguiente modelo matemático que integra la eficacia de agrupamiento $(\Gamma)$ y la eficiencia de la línea $(\Gamma)$ en una sola función objetivo que busca maximizar. Los valores que puede tomar esta función están entre cero y uno $[0,1]$, donde los valores iguales o cercanos a uno corresponden a los mejores diseños que maximizan el agrupamiento y el balanceo de las cargas de trabajo, mientras que los valores 
cercanos a cero son indeseables. A continuación se presenta la formulación matemática del modelo propuesto:

Maximizar:

$$
f=\Gamma_{\eta}
$$

Sujeto a:

$$
\begin{aligned}
& \sum_{k=1}^{m c} X_{i k}=1, \quad \forall \boldsymbol{i} \\
& \sum_{k=1}^{m c} Y_{j k}=1, \quad \forall \boldsymbol{j} \\
& Z_{i} \geq 1, \quad \forall \boldsymbol{i}
\end{aligned}
$$

Donde:

$\Gamma=$ Eficacia de agrupamiento

$$
\begin{aligned}
& \Gamma=\frac{\sum_{k=1}^{m c} \sum_{j=1}^{n} \sum_{i=1}^{m} Y_{j k} X_{i k} a_{i j}}{\sum_{j=1}^{n} \sum_{i=1}^{m} a_{i j}+\sum_{k=1}^{m c}\left[\left(\sum_{j=1}^{n} Y_{j k}\right)\left(\sum_{i=1}^{m} X_{i k}\right)\right]-\sum_{k=1}^{m c} \sum_{j=1}^{n} \sum_{i=1}^{m} Y_{j k} X_{i k} a_{i j}} \\
& \eta=E f i c i e n c i a \text { de la línea }
\end{aligned}
$$

$$
\eta=\sum_{j=1}^{n}\left[a_{i j}\left(\frac{1}{1+\left|t c_{j}-\sum_{i=1}^{m} \frac{t o p_{i j}}{Z_{i}}\right|}\right)\right]
$$

i=índice de las máquinas; $i=1, \ldots, m$ número de máquinas

j=índice de las partes; $j=1, \ldots, n$ número de partes

$k=$ índice de las celdas; $k=1, \ldots, \mathrm{mc}$ máximo número de celdas

$X_{i k}=1$, si la máquina i pertenece a la celda $k$, en caso contrario $X_{i k}=0$

$Y_{j k}=1$, si la parte j pertenece a la celda $k$, en caso contrario $Y_{j k}=0$

$Z_{i}=$ cantidad de máquinas del tipo $i$

$a_{i j}=1$, si la parte $j$ es procesada por la máquina i, en otro caso $a i j=0$ 
top $_{i j}=$ tiempo de operación de la parte $j$ en la máquina $i$ $t c_{j}=$ tiempo ciclo de la parte $j$

El modelo matemático toma en consideración que cada celda de manufactura se configura como una línea de ensamble lo cual se expresa en la función objetivo implementada (ecuación 1), en el producto de la eficacia de agrupamiento $\Gamma$ ) (ecuación 5) y la eficiencia de la línea de ensamble (ๆ) (ecuación 6), lo cual genera una relación directa entre las dos, que permite guiar el proceso de optimización hacia una solución óptima para ambas.

Las variables de decisión de este modelo son $X_{i k^{\prime}} Y_{j k}, Z_{i^{\prime}} X_{i k}$ $y Y_{j k}$ y de tipo binario; permiten respectivamente la asignación de las máquinas $(i)$ a las celdas $(k)$ y de las partes $(j)$ a las familias y/o celdas $(k) . Z_{i}$; es de tipo entero y representa la cantidad de máquinas $(i)$, con las cuales contará el sistema de manufactura.

La función objetivo está sujeta a tres restricciones: la primera (ecuación 2 ) es una restricción de unicidad que garantiza que cada máquina únicamente sea asignada a una celda; la segunda (ecuación 3) también es una restricción de unicidad que garantiza que cada parte únicamente sea asignada a una familia de partes y/o celda; y la tercera restricción permite lograr que el número mínimo de máquinas de cada tipo sea al menos uno (1) (ecuación 4).

Las características de cada sistema se ven definidas por la matriz de incidencia $\left(a_{i j}\right)$, la cual es una matriz binaria que establece si una parte es procesada en una máquina o no; la matriz de tiempos de operación $\left(\right.$ top $\left._{i j}\right)$ contiene los tiempos de las operaciones que se les realizan a las partes $(j)$ en las máquinas (i); y la matriz de tiempo ciclo $\left(t c_{j}\right)$ define el tiempo ciclo para cada parte. 


\section{Algoritmo genético (AG)}

a amplia aplicabilidad de los Algoritmos Genéticos (AG) en _problemas del mundo real se debe a que son adecuados para establecer soluciones en espacios de búsqueda de gran tamaño, dada su simplicidad conceptual. Estos fueron introducidos por Holland (1975) y se trata de algoritmos de búsqueda basados en la mecánica de la selección natural y la genética; se han aplicado en diversos campos tales como las matemáticas, ingeniería, biología y ciencias sociales (Goldberg, 1989). En los AG se combina el concepto de supervivencia del más apto con una estructura de intercambio de información al azar para buscar una solución adecuada a un problema de optimización.

Su proceso inicia generando una población inicial de posibles soluciones -individuos o cromosomas-; posteriormente, se evalúa la adaptación de cada uno de los individuos de la población y se seleccionan algunos de ellos para ser padres y reproducirse a través de un operador de cruce. Adicionalmente, se emplea un operador de mutación que modifica al azar la composición genética de algunos individuos con el fin de mejorar la diversidad de la población. Los procesos de evaluación, selección, reproducción y mutación se repiten hasta que se alcanza el criterio de terminación. El algoritmo genético implementado en este trabajo tiene la estructura que se presenta en pseudocódigo (Figura 1). 
Figura 1 Pseudocódigo general del Algoritmo Genético (AG)

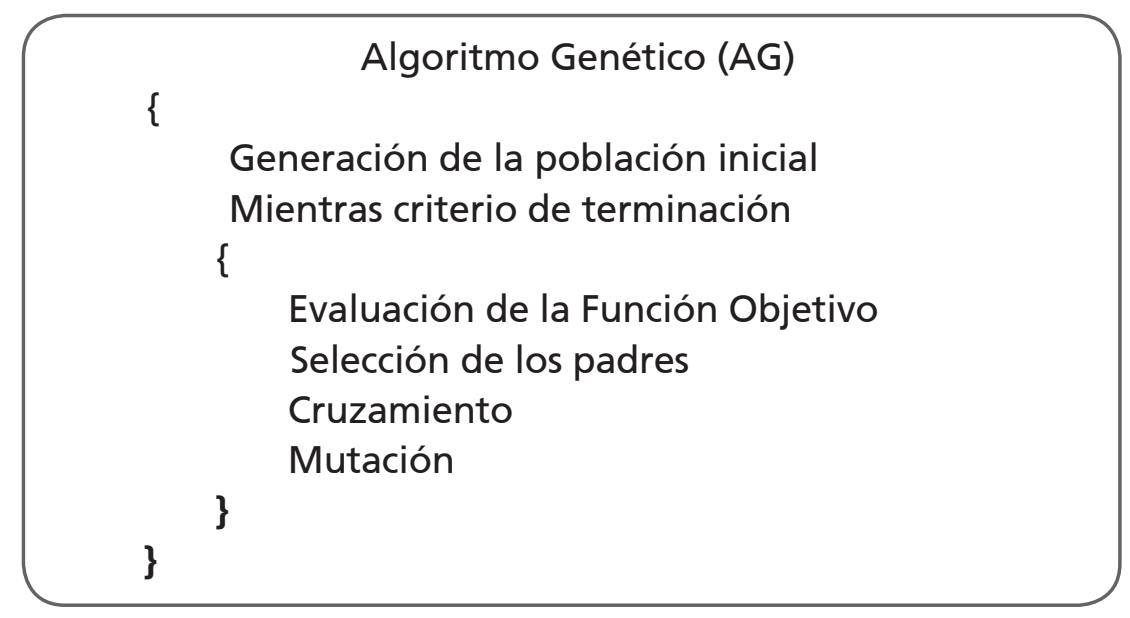

Fuente. Elaboración propia de los autores.

El algoritmo implementado inicia generando de forma aleatoria los cromosomas que representan los individuos de la población. A continuación se verifica la condición de terminación que, en nuestro caso, consiste en alcanzar un número de generaciones dado (100). De manera iterativa, el algoritmo ejecuta las siguientes funciones:

- Evaluación de la función fitness que corresponde a una de las métricas de agrupamiento.

- Selección de padres, la cual tiene dos fases. En la primera, se selecciona la élite -método de elitismo-, la cual corresponde al mejor $20 \%$ de los individuos de la población que se van a reproducir; adicionalmente, estos individuos no se reemplazan por cruzamiento o mutación mientras sigan perteneciendo a la élite. La segunda fase corresponde a la selección de los individuos para la reproducción; este proceso se realiza por torneo, en el cual se escogen al azar de entre la población un conjunto de individuos los cuales compiten entre sí y dependiendo de su adaptación se escoge al mejor - 
en este caso al mayor porque el fitness es una maximizaciónel cual pasa a conformar la población que posiblemente se va reproducir por cruzamiento.

- Cruzamiento entre padres. En esta función se seleccionan al azar los padres que se cruzan para formar dos hijos que reemplazan a los padres en la población si estos no pertenecen a la élite.

- Mutación de hijos. En esta función se verifica de forma aleatoria la aplicación de este operador a un individuo determinado siempre que no pertenezca a la élite y que obtenga una probabilidad inferior a la probabilidad de mutación establecida.

Es importante resaltar que el desempeño de un Algoritmo Genético depende en gran medida del método de codificación de las variables y/o soluciones candidatas, los operadores de cruce y mutación, los valores de los parámetros y de la función objetivo. Debido a esto a continuación se explica la codificación de las variables y los operadores de generación de la población inicial, cruzamiento y mutación.

\subsection{Codificación de las variables}

La codificación de las variables o soluciones candidatas es el proceso mediante el cual se le asigna una estructura de datos a las variables del modelo matemático para que puedan ser operadas por el proceso de optimización. En el caso de las variables $X_{i k} y Y_{j k}$ se empleó una codificación basada en grupo tal como es descrita por Gen, Lin, \& Zhang (2009). En la codificación implementada cada solución es representada con tres cromosomas, uno para cada variable de decisión $\left(X_{i k^{\prime}}, Y_{j k} y Z_{i}\right)$. Para el cromosoma $X_{i k}$ cada gen corresponde a una máquina y el valor de cada alelo de los genes -el valor almacenado en cada posición- corresponde 
al número de la celda a la cual se asigna la máquina (Figura 2 y Tabla 1). Para el cromosoma $Y_{j k}$ cada gen corresponde a una parte, y el valor de cada alelo de los genes -el valor almacenado en cada posición-corresponde al número de la familia a la cual se asigna la parte (Figura 2 y Tabla 1). La anterior codificación de las variables $X_{i k}$ y $Y_{j k^{\prime}}$ permite garantizar en la codificación el cumplimiento de restricciones descritas en las ecuaciones 2 y 3. En el caso de $Z_{i}$ cada gen corresponde a una máquina y el alelo corresponde a la cantidad de máquinas de cada tipo (Figura 2 y Tabla 1).

Figura 2 Ejemplo codificación de las variables

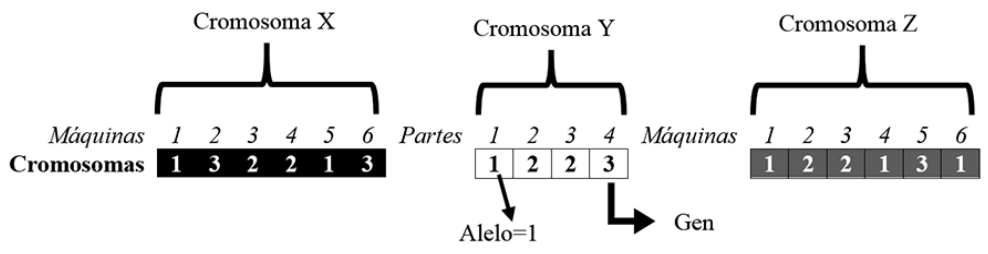

Para el ejemplo anterior (Figura 2) las celdas y familias de partes quedarían conformadas como se muestra a continuación (Tabla 1).

Tabla 1 Decodificación del ejemplo de la Figura 2

\begin{tabular}{|l|l|l|}
\hline \multicolumn{1}{|c|}{ Gen $\mathbf{X}$} & \multicolumn{1}{|c|}{ Gen $\mathbf{Y}$} & \multicolumn{1}{c|}{ Gen Z } \\
\hline $\begin{array}{l}\text { Celda 1= Máquinas } \\
\text { 1 y } 5\end{array}$ & Familia 1= Parte 1 & $\begin{array}{l}1 \text { máquina del tipo 1 } \\
\text { 3 máquinas del tipo 5 }\end{array}$ \\
\hline $\begin{array}{l}\text { Celda 2= Máquinas } \\
\text { 3 y 4 }\end{array}$ & Familia 2= Partes 2 y 3 & $\begin{array}{l}2 \text { máquinas del tipo 3 } \\
1 \text { máquina del tipo 4 }\end{array}$ \\
\hline $\begin{array}{l}\text { Celda 3= Máquinas } \\
\text { 2 y } 6\end{array}$ & Familia 3= Parte 4 & $\begin{array}{l}\text { 2 máquinas del tipo 2 } \\
1 \text { máquina del tipo 6 }\end{array}$ \\
\hline
\end{tabular}

Fuente. Elaboración propia de los autores. 


\subsection{Generación de la población inicial}

Dadas las características de esta codificación, la población inicial se genera asignando un número aleatorio entero entre 1 y el máximo número de celdas (genes $\mathrm{X}$ y $\mathrm{Y}$ ) o máquinas (gen $\mathrm{Z}$ ) a cada uno de los alelos de los genes de cada cromosoma.

\subsection{Cruzamiento}

En el proceso de cruzamiento, se realiza cruzamiento simple con un solo punto de cruce, intercambiando los genes de los dos padres como se muestra a continuación (Figura 3).

Figura 3 Ejemplo del funcionamiento del operador de cruzamiento
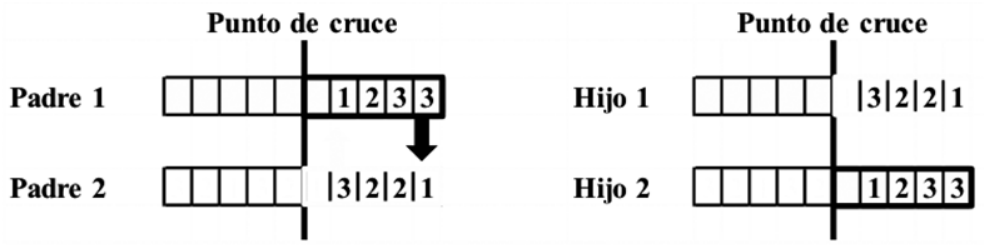

Fuente. Elaboración propia de los autores.

\subsection{Mutación}

La mutación se realiza a los alelos de los genes de forma aleatoria. Para cada alelo se genera un número aleatorio entre 0.0 y 1.0 que representa la probabilidad del alelo de ser mutado; si esta probabilidad es menor al porcentaje de mutación que se estableció, se genera aleatoriamente un número entre 1 y el máximo número de celdas, y se asigna al gen; en caso contrario no se modifica el gen. 


\section{Metodología y desarrollo experimental}

En términos generales, el proceso inicia con la formulación - matemática del modelo propuesto, el cual incluye la formación de las celdas de manufactura y el balanceo de las cargas de trabajo de cada una de las operaciones necesarias para fabricar las piezas. Posteriormente, se implementó el algoritmo genético considerando el modelo matemático propuesto, el cual define la codificación de las soluciones y el manejo de las restricciones que, en este caso, quedaron embebidas en la codificación. Luego, se ajustaron los parámetros del algoritmo genético para obtener la mejor configuración de este al resolver el modelo propuesto (Tabla 2); de esta forma, se garantiza que la evaluación del desempeño de la propuesta logre resultados confiables al resolver los problemas de prueba que se describen más adelante (Tabla 3).

Tabla 2. Parámetros del algoritmo genético implementado

\begin{tabular}{|l|l|}
\hline \multicolumn{1}{|c|}{ Parámetro } & \multicolumn{1}{c|}{ Valor } \\
\hline Tamaño de la población & 100 individuos \\
\hline Criterio de parada & 500 generaciones \\
\hline Porcentaje de la élite & $20 \%$ \\
\hline Probabilidad de Cruzamiento & 0.95 \\
\hline Probabilidad de Mutación & 0,05 \\
\hline
\end{tabular}

Fuente. Elaboración propia de los autores. 
Tabla 3 Problemas de prueba

\begin{tabular}{|c|l|}
\hline No. & \multicolumn{1}{|c|}{ Matriz de Incidencia } \\
\hline 1 & 4 máquinas - 5 partes (Kusiak, 1990) \\
\hline 2 & 4 máquinas - 5 partes (Yang \& Yang, 2008) \\
\hline 3 & 7 máquinas - 11 partes (Seifoddini \& Djassemi, 1996) \\
\hline 4 & 7 máquinas - 11 partes (Boctor, 1991) \\
\hline 5 y 6 & 15 máquinas - 15 partes (Yang \& Yang, 2008) \\
\hline
\end{tabular}

Fuente. Elaboración propia de los autores.

Cada uno de los problemas de prueba está compuesto por una matriz de incidencia, una matriz de tiempos de operación y un vector con los tiempos ciclo de cada parte. Las matrices de incidencia permiten evaluar la formación de las celdas y las familias de partes. Estos se obtuvieron de literatura especializada sobre el tema. Las matrices de los tiempos de operación de las partes y los tiempos ciclo de estas se generaron para cada una de las matrices de incidencia y poder evaluar el balanceo de las cargas de trabajo. Con el ánimo de presentar la estructura de cada problema de prueba (Tabla 4; Tabla 5; Tabla 6), se presentan las matrices que conforman el problema de prueba 1 , los demás problemas de prueba se incluyen como anexos a este documento.

Tabla 4. Matriz de incidencia del problema de prueba 1

\begin{tabular}{|c|c|c|c|c|c|c|}
\hline \multirow{6}{*}{$\begin{array}{l}\frac{n}{\pi} \\
\frac{5}{5} \\
\frac{5}{5} \\
\frac{\pi}{\Sigma} \\
\sum\end{array}$} & \multicolumn{6}{|c|}{ Partes } \\
\hline & & 1 & 2 & 3 & 4 & 5 \\
\hline & 1 & 0 & 1 & 0 & 1 & 1 \\
\hline & 2 & 1 & 0 & 1 & 0 & 0 \\
\hline & 3 & 0 & 1 & 0 & 1 & 0 \\
\hline & 4 & 1 & 0 & 1 & 0 & 0 \\
\hline
\end{tabular}

Fuente. Kusiak, (1990). 
Tabla 5. Matriz de tiempos de procesamiento del problema de prueba 1

\begin{tabular}{|c|c|c|c|c|c|c|}
\hline \multirow{6}{*}{ 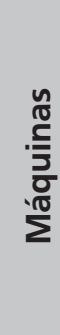 } & & \multicolumn{5}{|c|}{ Partes } \\
\hline & & 1 & 2 & 3 & 4 & 5 \\
\hline & 1 & & 30 & & 30 & 60 \\
\hline & 2 & 20 & & 20 & & \\
\hline & 3 & & 60 & & 60 & \\
\hline & 4 & 10 & & 10 & & \\
\hline
\end{tabular}

Fuente. Kusiak, (1990).

Tabla 6. Vector de tiempos ciclo del problema de prueba 1

\begin{tabular}{|l|c|c|c|c|c|}
\hline \multirow{2}{*}{} & \multicolumn{5}{|c|}{ Partes } \\
\cline { 2 - 6 } & $\mathbf{1}$ & $\mathbf{2}$ & $\mathbf{3}$ & $\mathbf{4}$ & $\mathbf{5}$ \\
\hline Tiempo ciclo & 10 & 30 & 10 & 30 & 60 \\
\hline
\end{tabular}

Fuente. Kusiak, (1990).

El proceso experimental llevado a cabo se enfoca en la evaluación del desempeño de la solución propuesta respecto a la obtención de soluciones óptimas, para lo cual se considera el promedio de la función objetivo de las soluciones obtenidas de cada problema de prueba y la desviación porcentual de las soluciones obtenidas con relación a la solución óptima en cada problema de prueba.

Dado lo anterior y con el ánimo de lograr resultados confiables, se determinó el número de réplicas necesario para las anteriores variables considerando una confiabilidad del $95 \%$ y un error del $5 \%$. Los resultados obtenidos son los siguientes: para el promedio de la función objetivo de las soluciones obtenidas, el número mínimo de réplicas fue de 288.77 y para la desviación porcentual de la función objetivo respecto a la solución óptima, el número de réplicas calculado fue 405. Debido a esto se realizaron 500 réplicas para garantizar la confiabilidad de los resultados. 
Adicionalmente, se registra el tiempo promedio requerido para obtener la mejor solución en cada réplica para orientar trabajos futuros.

\section{Resultados de discusión}

os resultados obtenidos del proceso experimental se presentan más adelante (Tabla 7). Allí, se puede apreciar para cada uno de los problemas de prueba el valor de la solución óptima, la mejor solución obtenida, el promedio de las soluciones obtenidas, la peor solución obtenida y el porcentaje promedio de desviación de las soluciones obtenidas con respecto a la solución óptima.

Los valores presentados representan resultados satisfactorios del proceso realizado, ya que demuestran que la aplicación de Algoritmos Genéticos a la solución simultánea de la formación de las celdas de manufactura y el balanceo de las cargas de trabajo, permiten obtener soluciones muy buenas que pueden impactar positivamente un sistema de producción al ser implementadas. Además, es importante resaltar que el promedio de las soluciones obtenidas es muy cercano al valor de la solución óptima de cada problema y la desviación porcentual promedio de las soluciones con respecto al óptimo es inferior al 1,2\%, por lo tanto, aun cuando se obtuvieran soluciones subóptimas, estas también representarían condiciones de operación que pueden constituir mejoras significativas al sistema. 
Tabla 7. Valores de la función objetivo obtenidos del proceso experimental

\begin{tabular}{|c|l|l|l|l|l|}
\hline $\begin{array}{c}\text { Problema } \\
\text { de } \\
\text { prueba }\end{array}$ & $\begin{array}{c}\text { Valor } \\
\text { óptimo }\end{array}$ & $\begin{array}{c}\text { Mejor } \\
\text { solución } \\
\text { obtenida }\end{array}$ & $\begin{array}{c}\text { Promedio } \\
\text { de las } \\
\text { soluciones } \\
\text { obtenidas }\end{array}$ & $\begin{array}{c}\text { Porcentaje } \\
\text { promedio } \\
\text { de } \\
\text { solución } \\
\text { obtenida }\end{array}$ & $\begin{array}{c}\text { desviación } \\
\text { de las } \\
\text { soluciones } \\
\text { respecto al } \\
\text { óptimo }\end{array}$ \\
\hline 1 & 0,9000 & 0,9000 & 0,9000 & 0,9000 & $0,00 \%$ \\
\hline 2 & 0,7188 & 0,7188 & 0,7188 & 0,7188 & $0,00 \%$ \\
\hline 3 & 1,0000 & 1,0000 & 0,9928 & 0,8400 & $0,72 \%$ \\
\hline 4 & 0,5150 & 0,5150 & 0,5090 & 0,4847 & $1,17 \%$ \\
\hline 5 & 0,5214 & 0,5214 & 0,5194 & 0,3020 & $0,40 \%$ \\
\hline 6 & 0,6032 & 0,6032 & 0,5960 & 0,3971 & $1,19 \%$ \\
\hline
\end{tabular}

Fuente. Elaboración propia de los autores.

Figura 4. Porcentaje promedio de desviación de las soluciones respecto al óptimo para cada problema de prueba

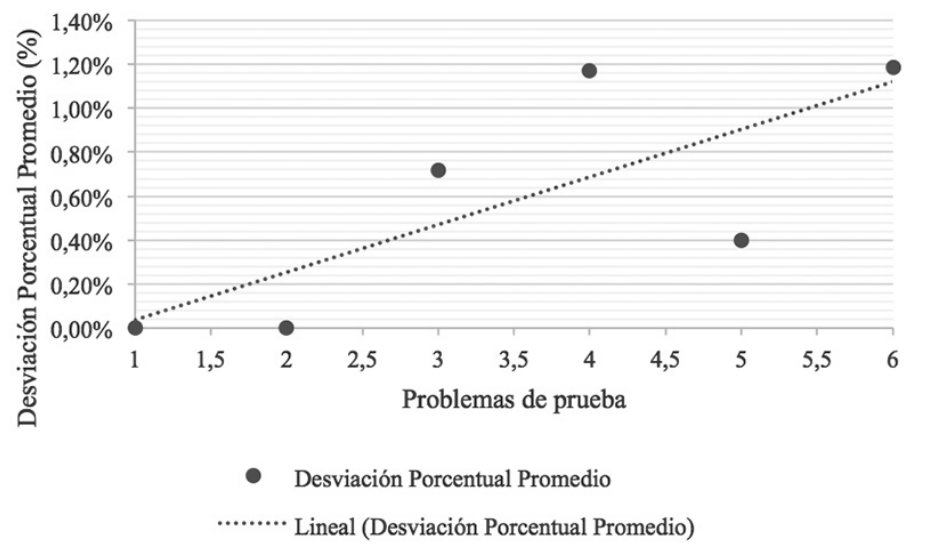


Figura 5. Tiempo promedio de las soluciones obtenidas para cada problema de prueba

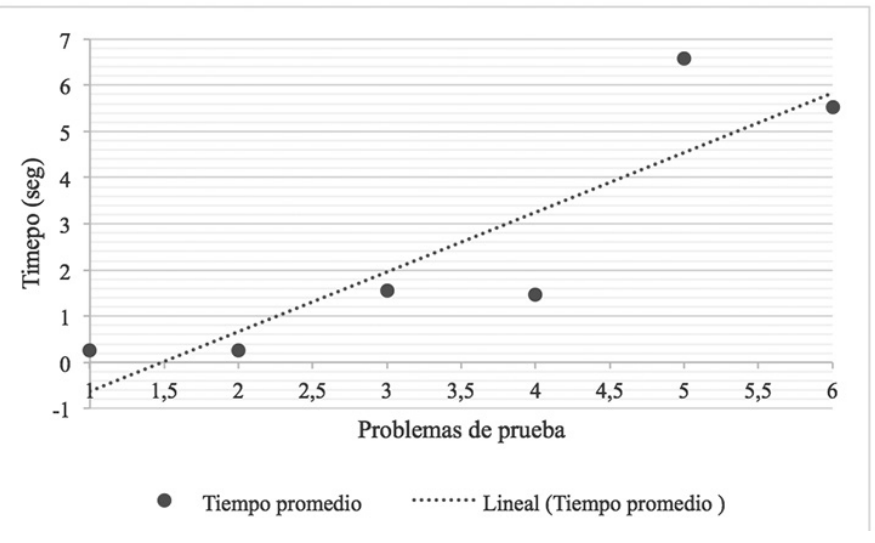

Fuente. Elaboración propia de los autores.

Al analizar el comportamiento de la desviación porcentual de las soluciones de los dos primeros problemas $(1,2)$, su valor es $0.0 \%$, por lo tanto, cada una de las réplicas de estos obtuvo la solución óptima. Además, al considerar la relación entre los problemas de prueba y la desviación porcentual de las soluciones, es posible evidenciar una tendencia creciente de esta en la medida que se incrementa el número de máquinas y partes de los problemas de prueba (Figura 4), que presenta adicionalmente una línea de tendencia que refuerza esta afirmación.

El tiempo promedio requerido para obtener la mejor solución de cada réplica también experimenta un comportamiento creciente (Figura 5). Sin embargo, la variación de este es mayor, ya que al considerar el valor de la desviación estándar con respecto al tiempo promedio, se puede afirmar que estos dos valores son en magnitud muy similares porque la diferencia entre ellos es muy baja (Tabla 8). Además, la diferencia entre el mayor y el menor tiempo obtenido es superior al promedio y este puede estar contenido varias veces en este rango. 
Aun cuando la variación en el tiempo de obtención de la mejor solución pueda variar, es necesario aclarar que los tiempos requeridos son pequeños, siendo el máximo valor no superior a 15 segundos, lo cual representa una ventaja en el proceso de diseño de sistemas de manufactura, ya que se pueden realizar varias réplicas en un corto tiempo lo que permitiría obtener diferentes alternativas factibles en un tiempo razonable.

Tabla 8. Valores de los tiempos requeridos para lograr las soluciones en el proceso experimental

\begin{tabular}{|c|c|c|c|c|}
\hline $\begin{array}{c}\text { Problema } \\
\text { de prueba }\end{array}$ & $\begin{array}{c}\text { Mayor } \\
\text { tiempo } \\
\text { obtenido }\end{array}$ & $\begin{array}{c}\text { Tiempo } \\
\text { promedio }\end{array}$ & $\begin{array}{c}\text { Menor } \\
\text { tiempo } \\
\text { obtenido }\end{array}$ & $\begin{array}{c}\text { Desviación } \\
\text { estándar } \\
\text { del tiempo }\end{array}$ \\
\hline 1 & 1,97 & 0,25 & 0,06 & 0,19 \\
\hline 2 & 1,37 & 0,25 & 0,05 & 0,17 \\
\hline 3 & 7,11 & 1,56 & 0,34 & 1,37 \\
\hline 4 & 7,38 & 1,46 & 0,31 & 1,44 \\
\hline 5 & 15,01 & 6,57 & 2,14 & 2,06 \\
\hline 6 & 14,82 & 5,53 & 2,04 & 2,25 \\
\hline
\end{tabular}

Fuente. Elaboración propia de los autores.

Por último, se analiza la convergencia de la propuesta de forma gráfica (Figura 6) que presenta la evolución de los valores de la función objetivo con respecto al tiempo de 10 réplicas de cada uno de los problemas de prueba. En cada imagen es evidente el mismo comportamiento; en primer lugar, una evolución rápida y creciente hacia el valor óptimo; y en segundo lugar, una dispersión entre los valores del tiempo requerido para lograr la mejor solución en cada caso. Adicionalmente, en la medida que se incrementa el tamaño del problema -número de máquinas y partes-, se evidencia el incremento de soluciones que alcanzan el valor óptimo. 
Figura 6. Comparación de la evolución de los valores de la función objetivo respecto al tiempo para 10 réplicas de los problemas de prueba: a) 1, b) 2, c) 3, d) 4, e) 5 y f) 6

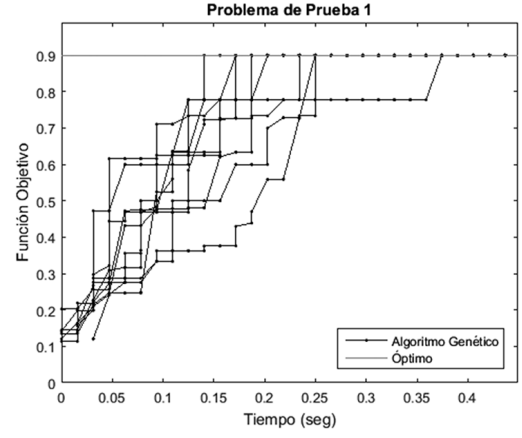

a)

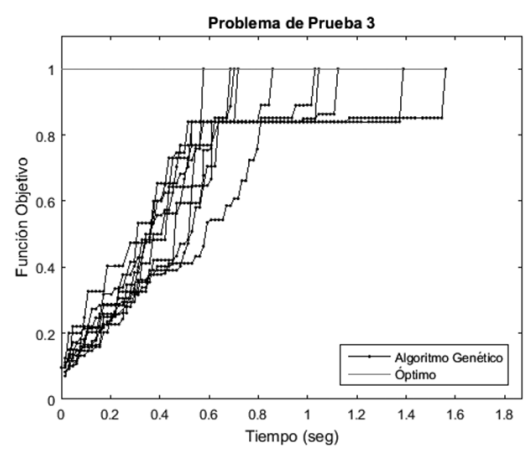

c)

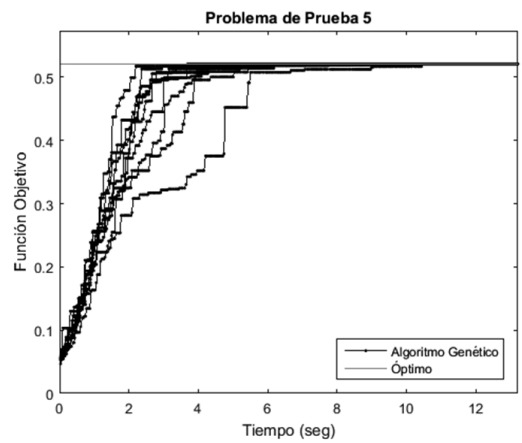

e)

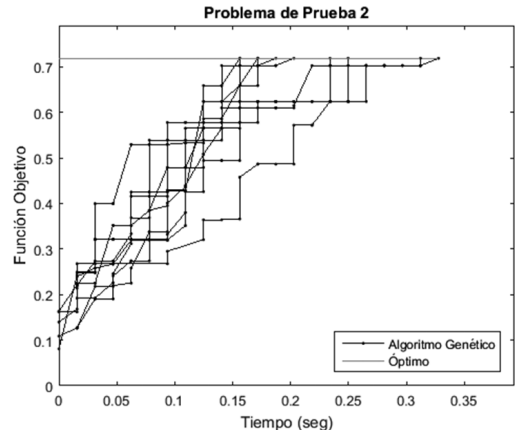

b)

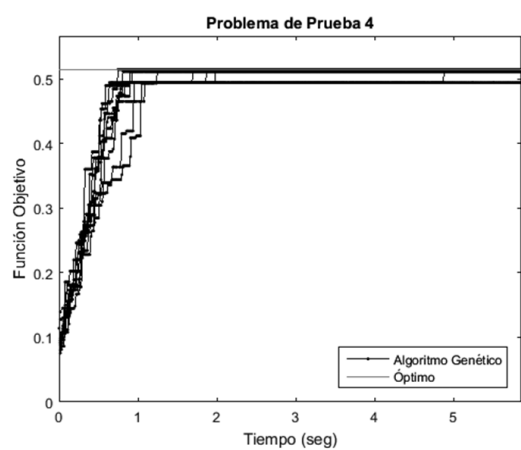

d)

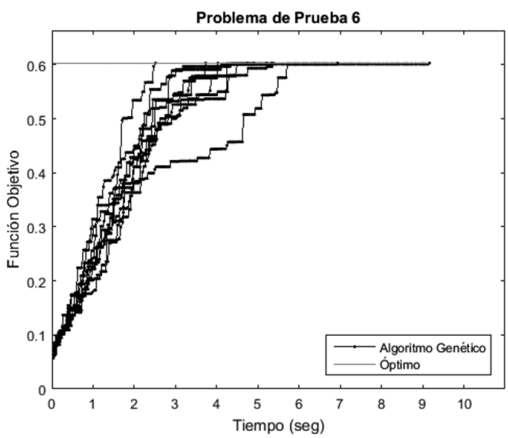

f) 


\section{Conclusiones}

A partir de los valores del porcentaje promedio de desviación de las soluciones respecto al óptimo, se puede afirmar que la solución simultánea de la formación de las celdas de manufactura y el balanceo de cargas de trabajo con Algoritmos Genéticos, es una alternativa viable que permite lograr muy buenas soluciones, con una desviación mínima de los valores óptimos. Ello representa en el ambiente industrial diseños de sistemas de manufactura más eficientes.

Al considerar el tiempo promedio en la obtención de las mejores soluciones, se puede evidenciar una clara ventaja para el proceso de diseño de sistemas de manufactura, ya que es posible realizar varias iteraciones que permiten validar los resultados, simularlos o realizar una evaluación más detallada de estos en un tiempo muy corto. Adicionalmente, si se realizan cambios en los productos o procesos que modifican la matriz de incidencia, los tiempos de operación y/o los tiempos ciclo, es posible en un corto tiempo formular nuevas propuestas para reconfigurar las celdas o modificar la asignación de las cargas de trabajo para lograr una mejor operación; esto influye positivamente en facilitar la flexibilidad del sistema. 


\section{Referencias bibliográficas}

Battaïa, O., \& Dolgui, A. (2013). A taxonomy of line balancing problems and their solutionapproaches. International Journal of Production Economics, 142(2), 259-277. http:// doi.org/10.1016/j.ijpe.2012.10.02.

Becker, C., \& Scholl, A. (2006). A survey on problems and methods in generalized assembly line balancing. European Journal of Operational Research, 168(3), 694-715. http:// doi.org/10.1016/j.ejor.2004.07.023.

Billo, R. E., Bidanda, B., \& Tate, D. (1996). A genetic cluster algorithm for the machine-component grouping problem. Journal of Intelligent Manufacturing. http://doi.org/10.1007/ BF00118082.

Boctor, F. F. (1991). A Jinear formulation of the machinepart cell formation problem. International Journal of Production Research, 29(2), 343-356. http://doi.org/ 10.1080/00207549108930075.

Gen, M., Lin, L., \& Zhang, H. (2009). Evolutionary techniques for optimization problems in integrated manufacturing system: State-of-the-art-survey. Computers and Industrial Engineering, 56(3), 779-808. http://doi.org/10.1016/j. cie.2008.09.034.

Goldberg, D. E. (1989). Genetic Algorithms in Search, Optimization and Machine Learning.

Holland, J. (1975). Adaptation in natural and artificial systems: an introductory analysis with applications to biology, control, and artificial intelligence. Ann Arbor: University of Michigan Press. 
Kusiak, A. (1990). Intelligent manufacturing systems. Prentice Hall.

Mejía, C., Lara, D. F., \& Nieto, C. (2010). Círculos tecnológicos de parentesco - Technological kinship circles. Revista Ingeniería E Investigación, 30(1), 163-167.

Papaioannou, G., \& Wilson, J. M. (2010). The evolution of cell formation problem methodologies based on recent studies (1997-2008): Review and directions for future research. European Journal of Operational Research, 206(3), 509521. http://doi.org/10.1016/j.ejor.2009.10.020

Rajagopalan, R., \& Fonseca, D. J. (2005). Manufacturing Cell Design through Genetic Algorithms. In IIE Annual Conference.Proceedings, pp. 1-6.

Romero, G. A., Mejía, C., \& Torres, J. A. (2015). Modelos matemáticos para la definición del layout de las celdas de manufactura. Revisión de literatura. Revista Tecnura, 19(46), 135-148.

Saeedi, S. (2010). Heuristic Approaches for Cell Formation in Cellular Manufacturing. Journal of Software Engineering and Applications, 03(07), 674-682. http://doi.org/10.4236/ jsea.2010.37077

Sarker, B. R. (2001). Measures of grouping efficiency in cellular manufacturing systems. European Journal of Operational Research, 130(3), 588-611. http://doi.org/10.1016/S03772217(99)00419-1

Seifoddini, H., \& Djassemi, M. (1996). A new grouping measure for evaluation of machine-component matrices. International Journal of Production Research, 34(5), 1179-1193. http:// doi.org/10.1080/00207549608904960 
Selim, H. M., Askin, R. G., \& Vakharia, A. J. (1998). Cell formation in group technology: Review, evaluation and directions for future research. Computers \& Industrial Engineering, 34(1), 3-20. http://doi.org/10.1016/\$0360-8352(97)00147-2

Spiliopoulos, K., \& Sofianopoulou, S. (2006). An efficient ant colony optimization system for the manufacturing cells formation problem. The International Journal of Advanced Manufacturing Technology, 36(5-6), 589-597. http://doi. org/10.1007/s00170-006-0862-2

Sundar, R., Balaji, a. N., \& Kumar, R. M. S. (2014). A Review on Lean Manufacturing Implementation Techniques. Procedia Engineering, 97, 1875-1885. http://doi.org/10.1016/j. proeng.2014.12.341

Suresh Kumar, C., \& Chandrasekharan, M. P. (1990). Grouping efficacy: a quantitative criterion for goodness of block diagonal forms of binary matrices in group technology. International Journal of Production Research, 28(2), 233243. http://doi.org/10.1080/00207549008942706

Venugopal, V., \& Narendran, T. T. (1992). A genetic algorithm approach to the machine-component grouping problem with multiple objectives. Computers \& Industrial Engineering, 22(4), 469-480. http://doi.org/10.1016/03608352(92)90022-C

Wu, T.-H., Chang, C.-C., \& Chung, S.-H. (2008). A simulated annealing algorithm for manufacturing cell formation problems. Expert Systems with Applications, 34(3), 16091617. http://doi.org/10.1016/j.eswa.2007.01.012

Yang, M. S., \& Yang, J. H. (2008). Machine-part cell formation in group technology using a modified ART1 method. European Journal of Operational Research, 188(1), 140152. http://doi.org/10.1016/j.ejor.2007.03.047 


\section{Anexo}

PROBLEMAS DE PRUEBA Y SOLUCIONES ÓPTIMAS

PROBLEMA DE PRUEBA 1

Matriz de tiempos por operación en segundos

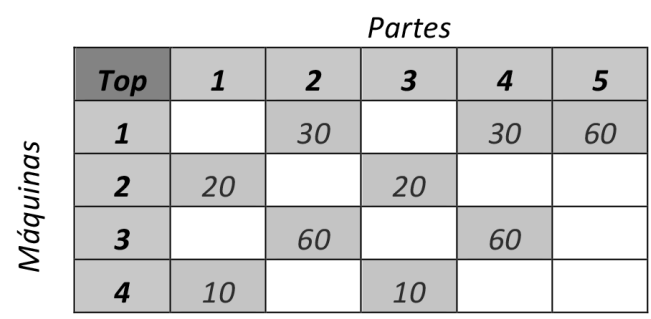

Tiempos ciclo por parte

\begin{tabular}{|c|c|c|c|c|c|}
\cline { 2 - 6 } \multicolumn{1}{c|}{} & \multicolumn{5}{c|}{ Partes } \\
\cline { 2 - 7 } \multicolumn{1}{c|}{} & $\mathbf{1}$ & $\mathbf{2}$ & $\mathbf{3}$ & $\mathbf{4}$ & $\mathbf{5}$ \\
\hline Tc & 10 & 30 & 10 & 30 & 60 \\
\hline
\end{tabular}

Soluciones óptimas

\begin{tabular}{|l|c|c|}
\hline & Máquinas & Partes \\
\hline Celda o Familia 1 & 2,4 & 1,3 \\
\hline Celda o Familia 2 & 1,3 & $2,4,5$ \\
\hline
\end{tabular}

\begin{tabular}{|c|c|c|c|c|}
\hline Máquinas & $\mathbf{1}$ & $\mathbf{2}$ & $\mathbf{3}$ & $\mathbf{4}$ \\
\hline$Z$ & 1 & 2 & 2 & 1 \\
\hline
\end{tabular}

PROBLEMA DE PRUEBA 2

Matriz de tiempos por operación en segundos

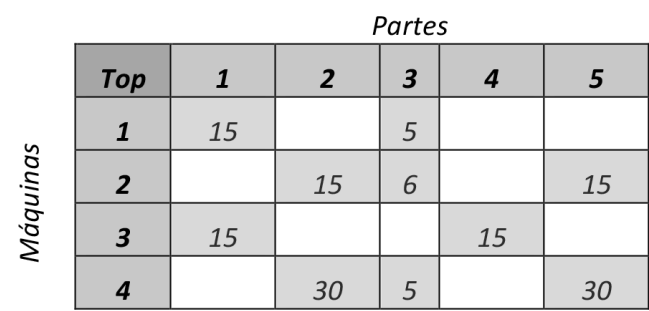

Tiempos ciclo por parte

\begin{tabular}{|c|c|c|c|c|c|}
\cline { 2 - 6 } \multicolumn{1}{c|}{} & \multicolumn{5}{c|}{ Partes } \\
\cline { 2 - 7 } \multicolumn{1}{c|}{} & $\mathbf{1}$ & $\mathbf{2}$ & $\mathbf{3}$ & $\mathbf{4}$ & $\mathbf{5}$ \\
\hline Tc & 15 & 15 & 5 & 15 & 15 \\
\hline
\end{tabular}


Edisson Suesca M./ Milena Bermúdez C./ Camilo Mejía M./ Luis Armando Cobo C./ Leonardo Rodríguez U.

\section{Soluciones óptimas}

\begin{tabular}{|l|c|c|}
\hline & Máquinas & Partes \\
\hline Celda o Familia 1 & 1,3 & 1,4 \\
\hline Celda o Familia 2 & 2,4 & $2,3,5$ \\
\hline
\end{tabular}

\begin{tabular}{|c|l|l|l|l|}
\hline Máquinas & $\mathbf{1}$ & $\mathbf{2}$ & $\mathbf{3}$ & $\mathbf{4}$ \\
\hline$Z$ & 1 & 1 & 1 & 2 \\
\hline
\end{tabular}

\section{PROBLEMA DE PRUEBA 3}

Matriz de tiempos por operación en segundos

\begin{tabular}{|c|c|c|c|c|c|c|c|c|c|c|c|}
\hline & & & & & & Parte & & & & & \\
\hline Top & 1 & 2 & 3 & 4 & 5 & 6 & 7 & 8 & 9 & 10 & 11 \\
\hline 1 & & & 20 & & & & 20 & & & & 20 \\
\hline 2 & 30 & 30 & & & & 30 & & & 30 & & \\
\hline 3 & 15 & 15 & & & & 15 & & & 15 & & \\
\hline 4 & & & & 18 & 18 & & & 18 & & 18 & \\
\hline 5 & & & 40 & & & & 40 & & & & 40 \\
\hline 6 & & & 60 & & & & 60 & & & & 60 \\
\hline 7 & & & & 36 & 36 & & & 36 & & 36 & \\
\hline
\end{tabular}

Tiempos ciclo por parte

\begin{tabular}{|c|c|c|c|c|c|c|c|c|c|c|c|}
\hline \multicolumn{10}{|c|}{} & \multicolumn{10}{c|}{ Partes } \\
\cline { 2 - 13 } & $\mathbf{1}$ & $\mathbf{2}$ & $\mathbf{3}$ & $\mathbf{4}$ & $\mathbf{5}$ & $\mathbf{6}$ & $\mathbf{7}$ & $\mathbf{8}$ & $\mathbf{9}$ & $\mathbf{1 0}$ & $\mathbf{1 1}$ \\
\hline Tc & 15 & 15 & 20 & 18 & 18 & 15 & 20 & 18 & 15 & 18 & 20 \\
\hline
\end{tabular}

Soluciones óptimas

\begin{tabular}{|l|c|c|}
\hline & Máquinas & Partes \\
\hline Celda o Familia 1 & 2,3 & $1,2,6,9$ \\
\hline Celda o Familia 2 & $1,5,6$ & $3,7,11$ \\
\hline Celda o Familia 3 & 4,7 & $4,5,8,10$ \\
\hline
\end{tabular}

\begin{tabular}{|c|l|l|l|l|l|l|l|}
\hline Máquinas & $\mathbf{1}$ & $\mathbf{2}$ & $\mathbf{3}$ & $\mathbf{4}$ & $\mathbf{5}$ & $\mathbf{6}$ & $\mathbf{7}$ \\
\hline$Z$ & 1 & 2 & 1 & 1 & 2 & 3 & 2 \\
\hline
\end{tabular}


PROBLEMA DE PRUEBA 4

Matriz de tiempos por operación en segundos

\begin{tabular}{|c|c|c|c|c|c|c|c|c|c|c|c|}
\hline & \multicolumn{11}{|c|}{ Partes } \\
\hline Top & 1 & 2 & 3 & 4 & 5 & 6 & 7 & 8 & 9 & 10 & 11 \\
\hline 1 & 10 & 10 & & & & 10 & & & & & \\
\hline 2 & & 13 & & & & 13 & & & 13 & & \\
\hline 3 & 25 & & 25 & & & & 25 & & & & 25 \\
\hline 4 & & & 16 & & & & 16 & & & & \\
\hline 5 & & & 13 & 13 & & & & & & & 13 \\
\hline 6 & & & & 25 & 25 & & & & & 25 & \\
\hline 7 & & & & & 8 & & & 8 & & 8 & \\
\hline
\end{tabular}

Tiempos ciclo por parte

\begin{tabular}{|c|c|c|c|c|c|c|c|c|c|c|c|}
\cline { 2 - 12 } \multicolumn{1}{c|}{} & \multicolumn{10}{c|}{ Partes } \\
\cline { 2 - 12 } & $\mathbf{1}$ & $\mathbf{2}$ & $\mathbf{3}$ & $\mathbf{4}$ & $\mathbf{5}$ & $\mathbf{6}$ & $\mathbf{7}$ & $\mathbf{8}$ & $\mathbf{9}$ & $\mathbf{1 0}$ & $\mathbf{1 1}$ \\
\hline Tc & 10 & 10 & 13 & 13 & 8 & 10 & 16 & 8 & 13 & 8 & 13 \\
\hline
\end{tabular}

Soluciones óptimas

\begin{tabular}{|l|c|c|}
\hline & Máquinas & Partes \\
\hline Celda o Familia 1 & 6,7 & $5,8,10$ \\
\hline Celda o Familia 2 & 1,2 & $1,2,6,9$ \\
\hline Celda o Familia 3 & $3,4,5$ & $3,7,11,4$ \\
\hline
\end{tabular}

\begin{tabular}{|c|l|l|l|l|l|l|l|}
\hline Máquinas & $\mathbf{1}$ & $\mathbf{2}$ & $\mathbf{3}$ & $\mathbf{4}$ & $\mathbf{5}$ & $\mathbf{6}$ & $\mathbf{7}$ \\
\hline Z & 1 & 1 & 2 & 1 & 1 & 3 & 1 \\
\hline
\end{tabular}


Edisson Suesca M./ Milena Bermúdez C./ Camilo Mejía M./ Luis Armando Cobo C./ Leonardo Rodríguez U.

PROBLEMA DE PRUEBA 5

Matriz de tiempos por operación en segundos

\begin{tabular}{|c|c|c|c|c|c|c|c|c|c|c|c|c|c|c|c|}
\hline & & & & & & & & Parte & & & & & & & \\
\hline Top & 1 & 2 & 3 & 4 & 5 & 6 & 7 & 8 & 9 & 10 & 11 & 12 & 13 & 14 & 15 \\
\hline 1 & & & & & & & & & & 7 & & 7 & & & 7 \\
\hline 2 & & 10 & 10 & & & & & & 10 & & & & & & \\
\hline 3 & 15 & & & & & & 15 & 15 & & & 15 & & & & \\
\hline 4 & & 30 & 30 & & & & & & 30 & & & & 30 & & \\
\hline 5 & & & & & 16 & 16 & & & & & & & & & \\
\hline 6 & & & & & & 23 & & & & & & & & 23 & \\
\hline 7 & & & & & 11 & 11 & & & & & & & & 11 & \\
\hline 8 & & 27 & 27 & & & & & & & & & & 27 & & \\
\hline 9 & & 13 & 13 & & & & & & 13 & & & & 13 & & \\
\hline 10 & & & & & & & & & & 25 & & 25 & & & \\
\hline 11 & & & & & 15 & & & & & & & & & 15 & \\
\hline 12 & & & 18 & & & & & & 18 & & & & 18 & & \\
\hline 13 & 23 & & & 23 & & & 23 & 23 & & & & & & & \\
\hline 14 & & & & & & & & & & 16 & & 16 & & & 16 \\
\hline 15 & & & & & 12 & 12 & & & & & & & & 12 & \\
\hline
\end{tabular}

Tiempos ciclo por parte

\begin{tabular}{|c|c|c|c|c|c|c|c|c|c|c|c|c|c|c|c|}
\hline & \multicolumn{15}{|c|}{ Partes } \\
\hline & 1 & 2 & 3 & 4 & 5 & 6 & 7 & 8 & 9 & 10 & 11 & 12 & 13 & 14 & 15 \\
\hline Tc & 15 & 10 & 10 & 23 & 11 & 11 & 15 & 15 & 10 & 7 & 15 & 7 & 13 & 11 & 7 \\
\hline
\end{tabular}

Soluciones óptimas

Soluciones óptimas
\begin{tabular}{|l|c|c|}
\hline & Máquinas & Partes \\
\hline Celda o Familia 1 & $2,4,8,9,12$ & $2,3,9,13$ \\
\hline Celda o Familia 2 & $5,6,7,11,15$ & $5,6,14$ \\
\hline Celda o Familia 3 & 3,13 & $1,4,7,8,11$ \\
\hline Celda o Familia 4 & $1,10,14$ & $10,12,15$ \\
\hline
\end{tabular}

\begin{tabular}{|l|l|l|l|l|l|l|l|l|l|l|l|l|l|l|l|}
\hline Máquinas & 1 & 2 & 3 & 4 & 5 & 6 & 7 & 8 & 9 & 10 & 11 & 12 & 13 & 14 & 15 \\
\hline
\end{tabular} 
Diseño de celdas de manufactura considerando el balanceo de las cargas de trabajo con algoritmos genéticos

PROBLEMA DE PRUEBA 6

Matriz de tiempos por operación en segundos

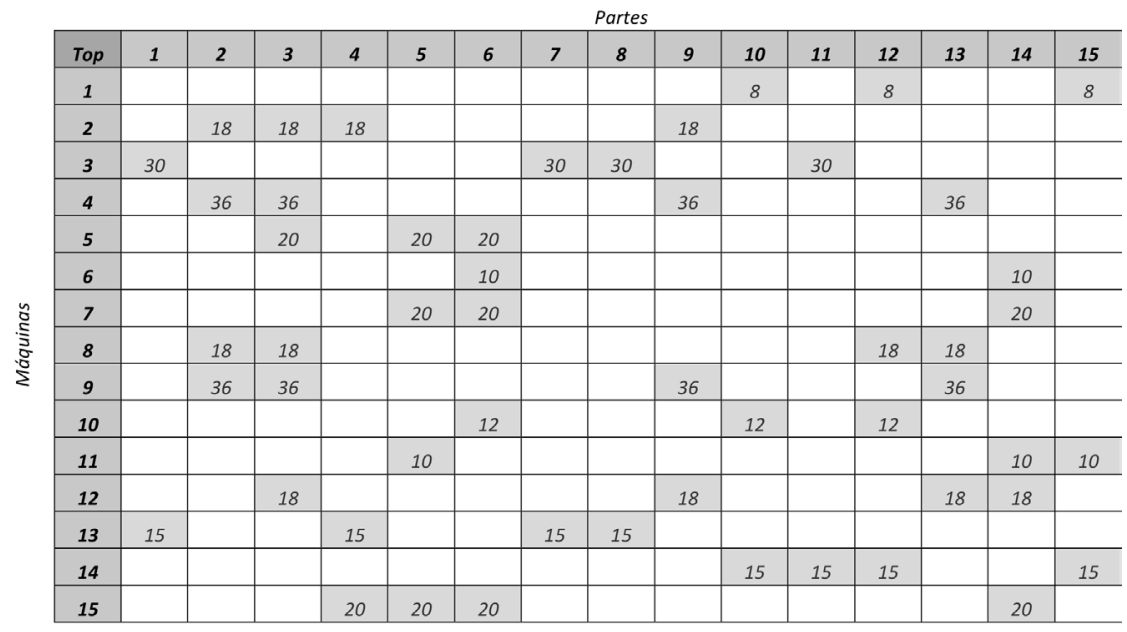

Tiempos ciclo por parte

\begin{tabular}{|c|c|c|c|c|c|c|c|c|c|c|c|c|c|c|c|}
\hline \multicolumn{16}{|c|}{ 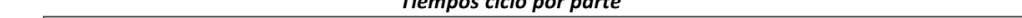 } \\
\hline & \multicolumn{15}{|c|}{ Partes } \\
\hline & 1 & 2 & 3 & 4 & 5 & 6 & 7 & 8 & 9 & 10 & 11 & 12 & 13 & 14 & 15 \\
\hline$T c$ & 15 & 18 & 18 & 15 & 10 & 10 & 15 & 15 & 18 & 8 & 15 & 8 & 18 & 10 & 8 \\
\hline
\end{tabular}

Soluciones óptimas

\begin{tabular}{|l|c|c|}
\hline & Máquinas & Partes \\
\hline Celda o Familia 1 & $2,4,8,9,12$ & $2,3,9,13$ \\
\hline Celda o Familia 2 & $5,6,7,11,15$ & $5,6,14$ \\
\hline Celda o Familia 3 & 3,13 & $1,4,7,8,11$ \\
\hline Celda o Familia 4 & $1,10,14$ & $10,12,15$ \\
\hline
\end{tabular}

\begin{tabular}{|l|l|l|l|l|l|l|l|l|l|l|l|l|l|l|l|}
\hline Máquinas & 1 & 2 & 3 & 4 & 5 & 6 & 7 & 8 & 9 & 10 & 11 & 12 & 13 & 14 & 15 \\
\hline
\end{tabular}

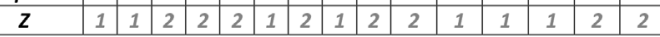

